\title{
Structural characterization of epitaxial YBCO thin films prepared by a fluorine-free sol-gel method for coated conductors
}

\author{
Jie Lian ${ }^{1}$, Haibo Yao $^{2}$, Donglu Shi ${ }^{3}$, Lumin Wang ${ }^{1}$, Yongli $\mathrm{Xu}^{3}$, \\ Qing $\mathrm{Liu}^{2}$ and $\mathbf{Z} \mathbf{H a n}^{2,4}$ \\ ${ }^{1}$ Department of Nuclear Engineering and Radiological Sciences, University of Michigan, \\ Ann Arbor, MI 48109, USA \\ ${ }^{2}$ Applied Superconductivity Research Center, Tsinghua University, Beijing, \\ People's Republic of China \\ ${ }^{3}$ Department of Chemical and Materials Engineering, University of Cincinnati, Cincinnati, \\ OH 45221, USA \\ ${ }^{4}$ Department of Materials Science and Engineering, Tsinghua University, Beijing, \\ People's Republic of China
}

Received 16 January 2003, in final form 11 April 2003

Published 13 June 2003

Online at stacks.iop.org/SUST/16/838

\begin{abstract}
Using a fluorine-free sol-gel method, both $c$ - and $a$-axis textured YBCO thin films were synthesized in low oxygen partial pressure. The experimental results of both $\mathrm{x}$-ray diffraction $2 \theta$ scan and pole figure showed well epitaxial grown films on $\mathrm{LaAlO}_{3}$ (LAO) single crystal substrates. When the processing condition was altered, the scanning electron microscopy indicated a large fraction of $a$-axis oriented grains in the film. High-resolution transmission electron microscopy was performed on both types of films and revealed interface lattice structural characteristics. A classic nucleation and growth model was used to explain the texturing mechanism. Other possible mechanisms for the $a$-axis oriented grains are also discussed.
\end{abstract}

\section{Introduction}

In previous studies, the synthesis of YBCO films through trifluoroacetate metalorganic deposition (TFA) [1,2] has been well established, this method can readily produce films with a high $J_{\mathrm{c}}$ well over $1 \mathrm{MA} \mathrm{cm}^{-2}$ at $77 \mathrm{~K}$ on both single crystal substrates [2-4] and buffered metallic substrates of RABiTS $[5,6]$. The TFA approach, in comparison with the vapour deposition methods including pulsed laser deposition (PLD), e-beam co-evaporation, sputtering and other popular physical methods, has demonstrated several advantages, particularly in industrial applications such as low cost, continuous process and easy scale-up. In early studies, the role of fluorine in the TFA method was believed to be associated with the carbon removal process [7-13]. Experimental results have shown that the transport properties could not be effectively enhanced due to the residuals of carbon in the films, particularly along the grain boundaries. Therefore, the transport $J_{\mathrm{c}}$ was low even for well-textured epitaxial YBCO films that did not contain fluorine. In our recent studies, however, using a fluorine-free sol-gel approach involving the trimethylacetate salts and proponic acid (TMAP) precursor solution, welltextured, epitaxial YBCO thin films were synthesized [1416]. The transport critical current density was found to steadily increase as the microstructure was improved in the YBCO thin film prepared by the fluorine-free sol-gel TMAP method. A high transport $J_{\mathrm{c}}$ was recently obtained on the order of $1 \mathrm{MA} \mathrm{cm}{ }^{-2}$ at $77 \mathrm{~K}$ [17]. Although the detailed mechanism on the carbon removal process has not yet been clearly established for the fluorine-free sol-gel synthesis, it provides an effective alternative method for making longlength conductors in large-scale applications. In recent studies, we have found that the TMAP approach presents several 
unique advantages including:

(1) no HF formed during the process, which is difficult to remove in the TFA method;

(2) stable precursor solution having a long shelf time of several months;

(3) much denser microstructure compared to TFA films;

(4) no extra phases (such as $\mathrm{BaF}_{2}$ in TFA approach), in addition to YBCO.

With these advantages, our current experimental results have clearly shown that high-quality YBCO thin films can be synthesized by the newly-developed, fluorine-free TMAP method for coated conductor development. The transport $J_{\mathrm{c}}$ is expected to improve upon the optimization of processing parameters and microstructures. For the fundamental understanding of the synthesis chemistry, it is of great interest to comparatively study the processing routes of both TFA and TMAP. In the past, the critical point in solution synthesis of YBCO film was focused on the removal of carbon. It was believed that carbon could be removed from the material at a low temperature $\left(<400{ }^{\circ} \mathrm{C}\right)$ at the burnout stage in wet oxygen, and fluorine could then be removed at a higher temperature $\left(>700{ }^{\circ} \mathrm{C}\right)$ in a humid and low oxygen partial pressure environment [1-3]. However, the results from the TMAP approach have indicated that the technique allows the use of a carbon containing precursor film to be reacted at $700-800{ }^{\circ} \mathrm{C}$ to obtain high- $J_{\mathrm{c}}$ films. This is achieved by specific processing conditions that allow for complete removal of carbon.

Other issues in this novel approach involve a detailed structural characterization of the epitaxial film and the study on growth mechanisms. In this paper, we present further experimental data on the film texturing mechanism and structural characterization based on high-resolution transmission electron microscopy (HRTEM). The mechanisms of YBCO grain formation are discussed.

\section{Experimental details}

Our fluorine-free sol-gel YBCO solutions were developed inhouse [14-16]. For the precursor solution, stoichiometric $(1: 2: 3)$ yttrium trimethylacetate, barium hydroxide and copper trimethylacetate powders were dissolved in a mixed propionic acid/amine solvent with an oxide concentration between 0.1 and $0.5 \mathrm{~mol}^{-1}$. The addition of amine was important because it greatly improved the solubility of the precursor powders in propionic acid. The stock solution was stable in air with a shelf-life longer than two years. Xylenes of alcohols were used for dilution and for controlling solution viscosity at $10-100 \mathrm{cP}$. The films on LAO were deposited by spin coating at $3000-3500 \mathrm{rpm}$ and were dried at $200-250^{\circ} \mathrm{C}$ for several minutes. This process was repeated to build up the desired film thickness $(0.5-0.6 \mu \mathrm{m})$. The LAO single crystals had dimensions of $12 \times 12 \times 1 \mathrm{~mm}^{3}$ and were purchased from MTI company. One side of the single crystal was polished as-purchased.

After spin coating, the samples were heat treated in a quartz furnace under the humid condition with $200 \mathrm{ppm}$ oxygen. The temperature was increased from room
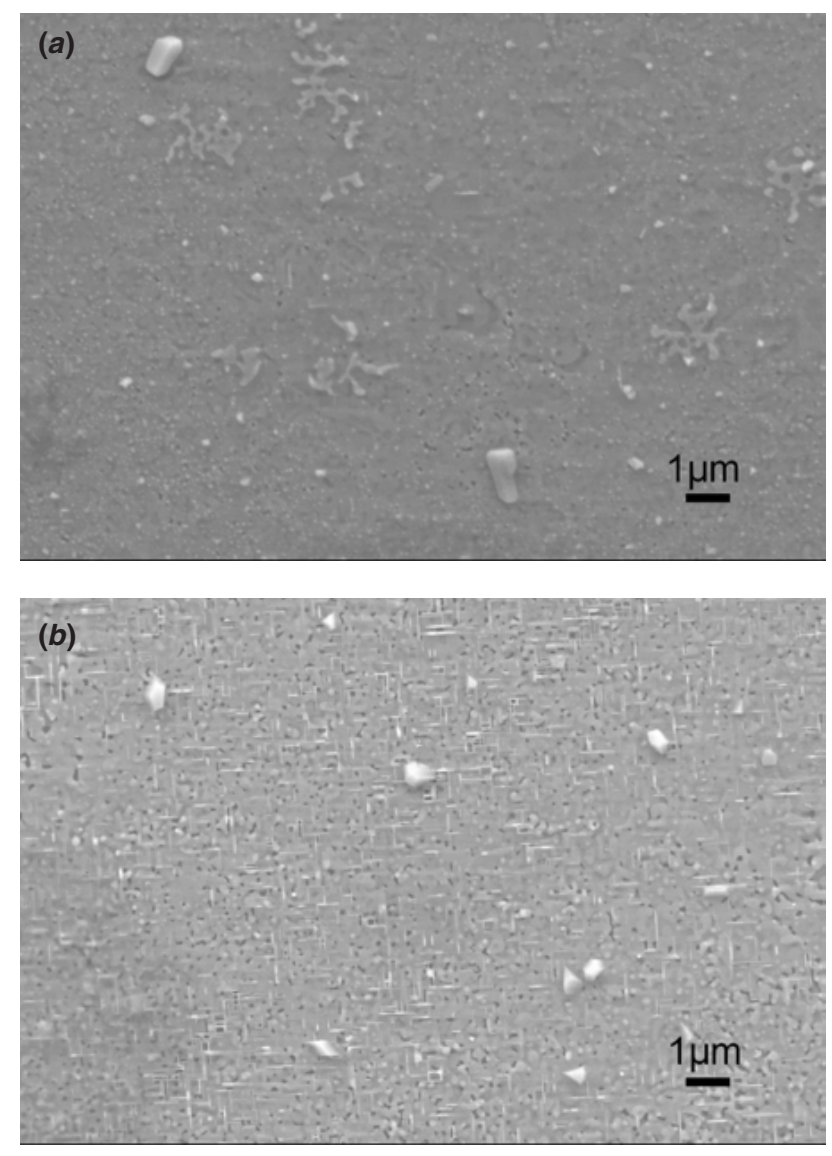

Figure 1. SEM photographs showing the surface morphologies of the $c$-axis $(a)$ and the $a$-axis $(b)$ oriented YBCO thin films.

temperature to $745{ }^{\circ} \mathrm{C}$ at the rate of $25{ }^{\circ} \mathrm{C} \mathrm{min}-1$, then to $750{ }^{\circ} \mathrm{C}$ at the rate of $1{ }^{\circ} \mathrm{C} \mathrm{min}-1$, dwelling at $750{ }^{\circ} \mathrm{C}$ for $80 \mathrm{~min}$. A higher maximum temperature up to $820^{\circ} \mathrm{C}$ was also used. Ten minutes before the end of dwelling process, a dry atmosphere was used. Then the temperature was decreased to $450{ }^{\circ} \mathrm{C}$ at the rate of $2.5^{\circ} \mathrm{C} \mathrm{min}-1$. As the temperature was lowered to $525^{\circ} \mathrm{C}$, the atmosphere of $200 \mathrm{ppm}$ oxygen was switched to pure oxygen, dwelling at $450{ }^{\circ} \mathrm{C}$ for $60 \mathrm{~min}$, then the samples were furnace-cooled to room temperature.

A Philips X-ray diffractometer with $\mathrm{Cu} \mathrm{K} \alpha$ radiation was used to carry out the texture investigations. The $\mathrm{x}$-ray wavelength was $1.54 \AA$ with a beam size of $3 \mathrm{~mm}$. Scanning electron microscopy (SEM) experiments were performed on a Hitachi 2000. Some specimens were also examined by scanning electron microscopy in a JEOL JSM 5400 microscope that was operated at $10 \mathrm{kV}$. No conductive coating was applied. The high-resolution TEM (HRTEM) experiments were performed on a JEOL 2010F TEM. Resistivity measurements were carried out down to liquidhelium temperature using a standard four-probe method. The transport $J_{\mathrm{c}}$ was determined with a voltage criterion of $10 \mu \mathrm{v} \mathrm{cm}$.

\section{Results and discussion}

Figure 1 shows the SEM micrographs of the YBCO thin film surface morphology. Figure 1(a) shows smooth $c$-axis oriented 


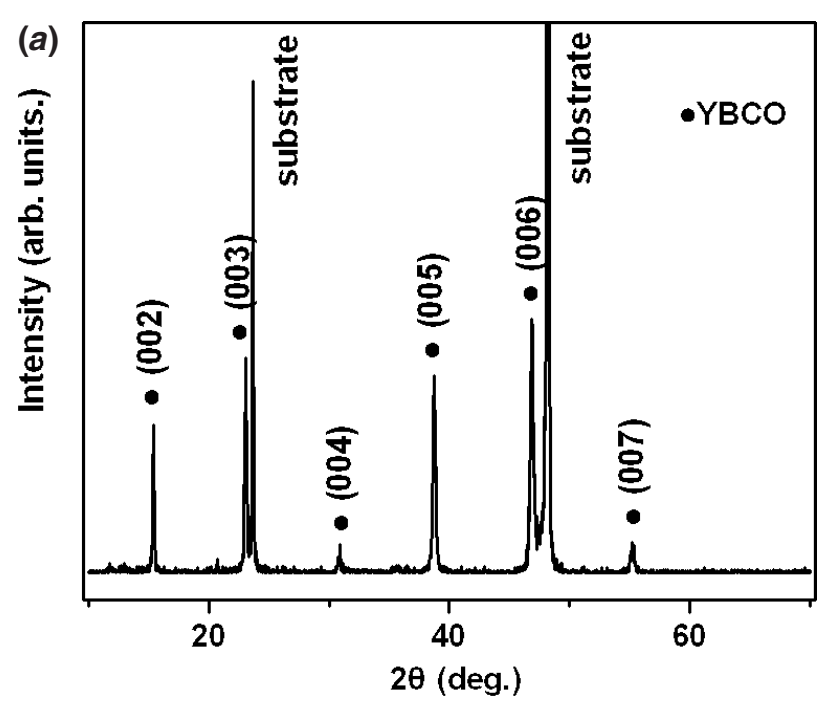

(b)

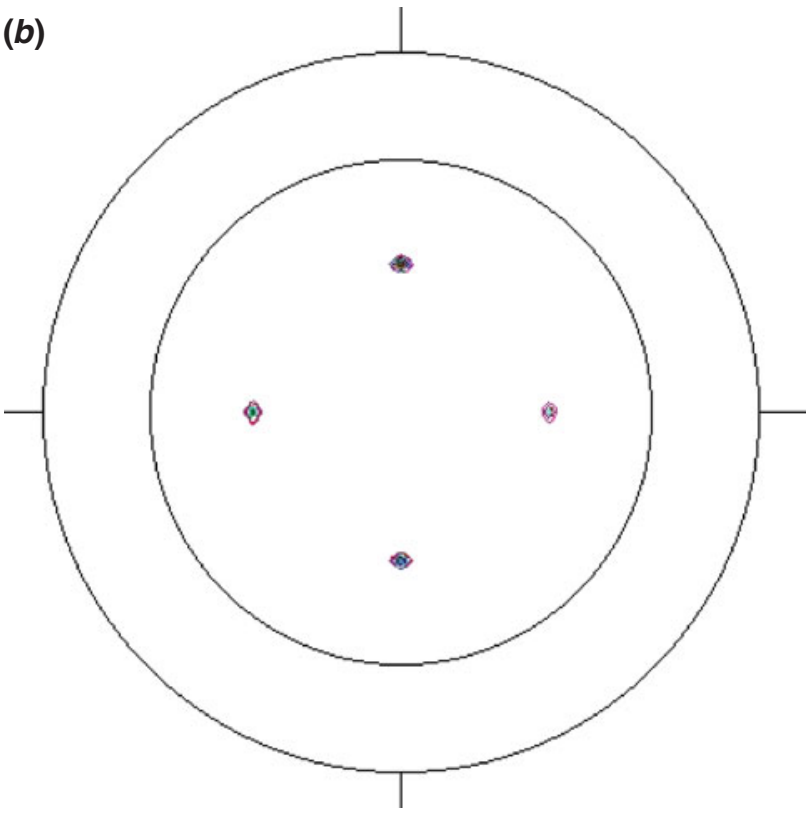

Figure 2. XRD $2 \theta$ scan $(a)$ and pole figure $(b)$ of the $c$-axis oriented YBCO thin film.

film $\left(780{ }^{\circ} \mathrm{C}, 200 \mathrm{ppm}\right)$, while figure $1(b)$ reveals a more $a$-axis textured film $\left(820^{\circ} \mathrm{C}, 200 \mathrm{ppm}\right)$. As can be seen in figure $1(a)$, the surface of the film is quite dense with a few particles that have been identified as a copper-rich phase by energy dispersive spectrum (EDS). In contrast, the surface morphology of the $a$-axis oriented film exhibits a 'grid' like feature as shown in figure $1(b)$. These structural characteristics can be best distinguished by $\mathrm{x}$-ray diffraction (XRD) analysis, especially in pole figure patterns. Figure $2(a)$ shows the XRD $2 \theta$ scan of the $c$-axis oriented YBCO film with all (001) peaks indicating a well-textured grain structure. The pole figure of the $c$-axis oriented YBCO film is shown in figure $2(b)$, which is consistent with the XRD $2 \theta$ scan.

To further study the epitaxial structure of the YBCO thin films, HRTEM was performed on both types of thin films. For the $c$-axis oriented thin film, the HRTEM revealed a coherent interface structure as shown in figure 3. In figure 3, the lattice image shows a clear lattice-matching interface

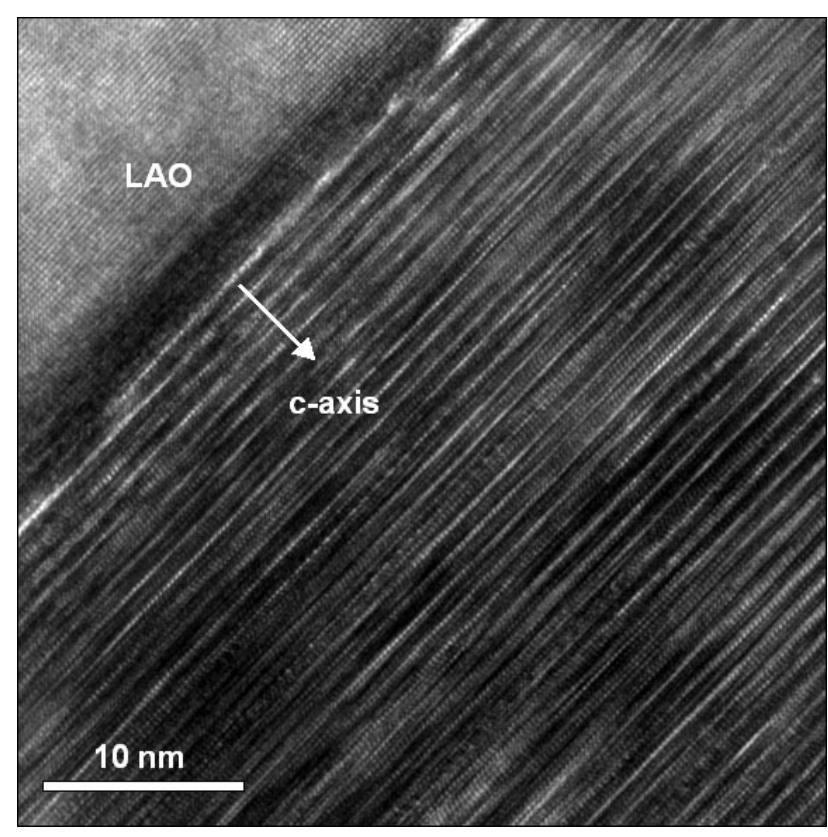

Figure 3. HRTEM image of the $c$-axis oriented YBCO thin film.

between the LAO substrate and the YBCO phase. It was found that this $c$-axis orientation extended all the way up to the film surface with a total thickness of $0.5 \mu \mathrm{m}$. However, in the $a$-axis oriented film, the interface structure exhibits entirely different feature as can be seen in figure 4 . Figure 4(a) shows an initial epitaxial growth at the interface that is interrupted at about $50 \mathrm{~nm}$ thickness by an $a$-axis growth. The transition of the $a$-axis growth appears to take place sharply with a clean interface between the $c$ - and $a$-axis grains. The similar YBCO grain interfaces are revealed at the same magnification in figure $4(b)$. In this figure, one can see a steplike interface between these two-types of grains. However, at a high magnification, more complex lattice structures are seen at the interface between the $c$-and $a$-axis oriented grains as shown in figure 4(c). In this figure, there appears to be a boundary of $4 \mathrm{~nm}$ width in which the lattices have gone through a transitional variation of $45^{\circ}$. The electron diffraction patterns for both $c$ - and $a$-axis orientations are shown in figure 5. As can be seen, both diffraction spots of substrate LAO and the YBCO grains are identified with distinct characteristics of $c$ - (figure 5(a)) and $a$-axis (figure $5(b)$ ) orientations.

Figure 6 shows the superconducting transitions by ac susceptibility (figure $6(a)$ ) and transport resistivity (figure $6(b)$ ) measurement for the $c$-axis oriented YBCO thin film. Quite sharp transitions take place at an onset value near $90 \mathrm{~K}$. The transport $J_{\mathrm{c}}$ has been reported previously in YBCO films prepared by the same method. A high value on the order of $10^{6} \mathrm{~A} \mathrm{~cm}^{-2}$ at $77 \mathrm{~K}$ and zero magnetic field has been achieved [17]. This value is expected to improve as the processing parameters are further optimized.

In the synthesis of TMAP YBCO films, the texturing mechanism is of great interest. As is shown above, both $c$ - and $a$-axis textured films are obtained due to a slight variation of processing conditions, majorly, the heat treatment temperature and oxygen partial pressure. The effect of oxygen partial pressure on the YBCO formation mechanism has been 

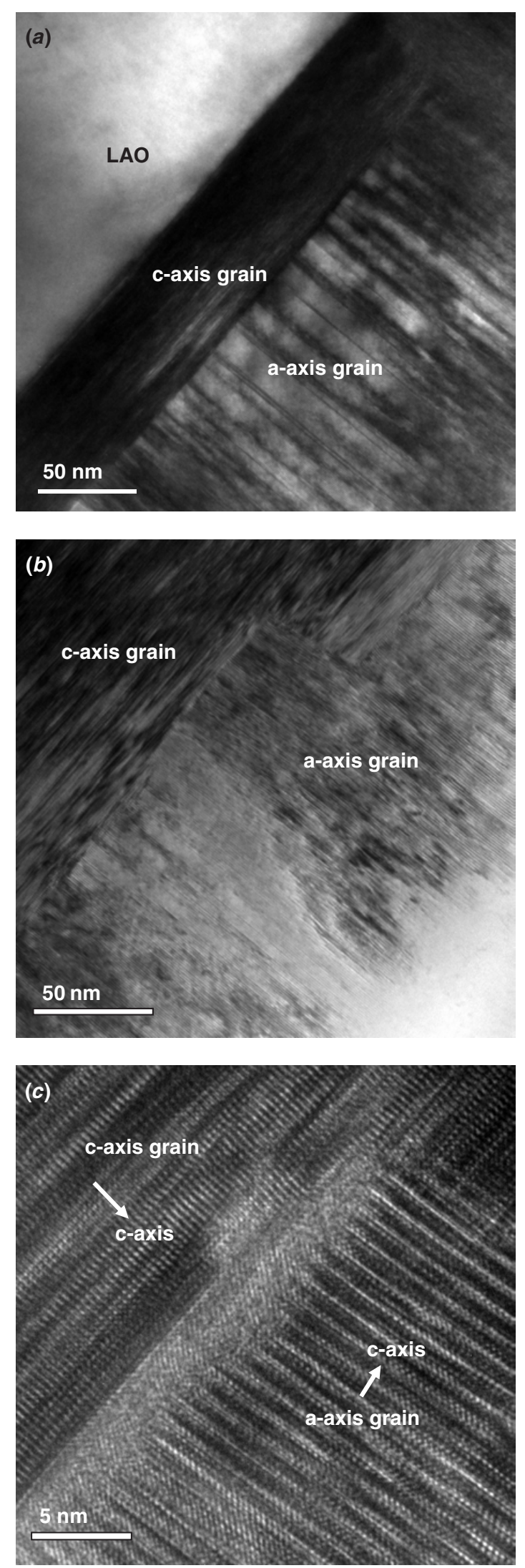

Figure 4. HRTEM images of the $a$-axis oriented YBCO film. (a) The interface image showing the interruption of the epitaxial growth by a abrupt a-growth, $(b)$ a step-like interface between the $c$ - and $a$-axis oriented grains and $(c)$ lattice image of a transitional boundary between the $c$-and $a$-axis oriented grains.
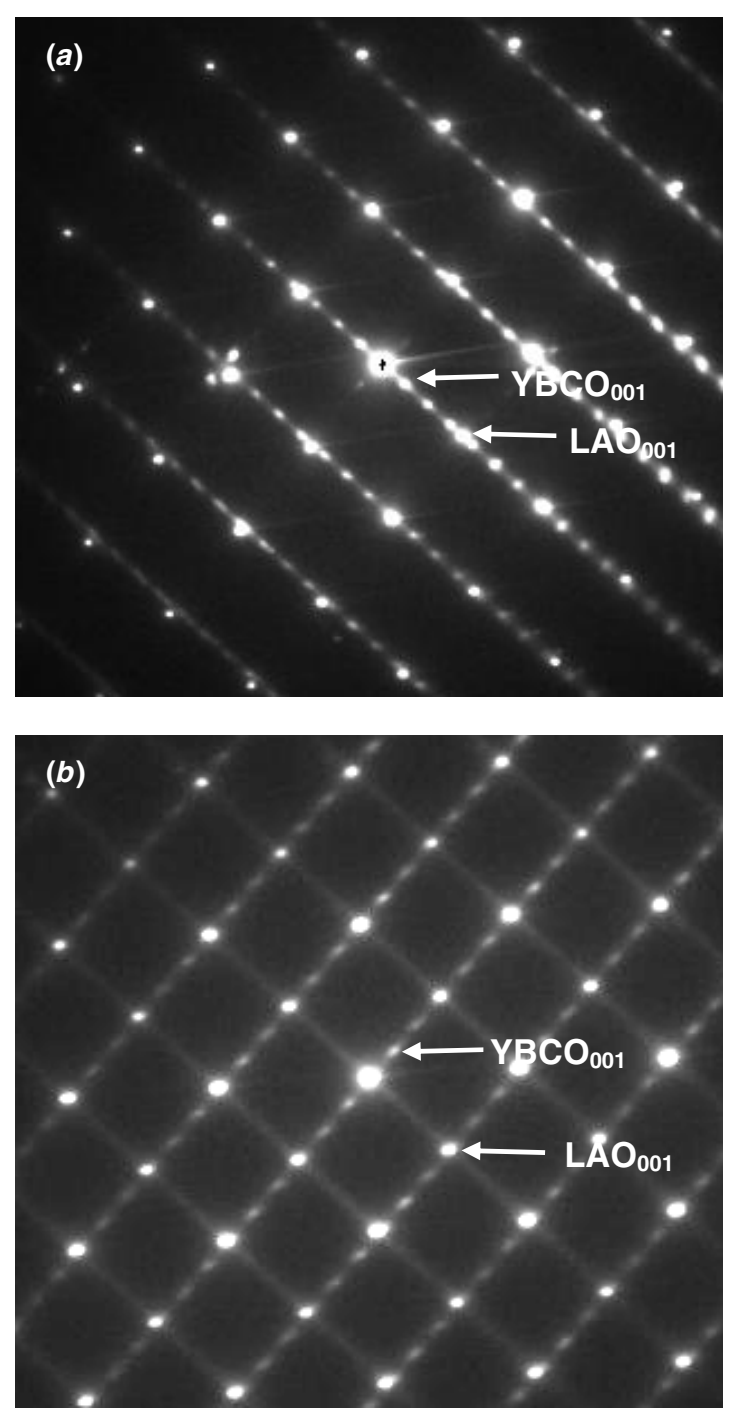

Figure 5. Selected area electron diffraction patterns of LAO substrate and (a) $c$-axis and $(b) a$-axis oriented YBCO thin films.

extensively studied for both bulk [18] and thin films [19, 20]. For the TFA synthesis of epitaxial YBCO films by postannealing, at a given temperature, lower oxygen partial pressure has been found to promote the $c$-axis epitaxy. This behaviour is accounted for by the enhanced oxygen-vacancyinduced cation diffusion process at low oxygen pressures. Later studies have shown that, below the critical oxygen partial pressure, the perovskite structure becomes unstable with respect to other phases, therefore setting a lower limit for the growth of YBCO. At various combinations of temperature and oxygen pressure, at low temperature limit, kinetics becomes important as the thermodynamic conditions favour the nonequilibrium phases, e.g., the amorphous structure. In previous studies, however, the discussion mainly focuses on the formation mechanisms of YBCO and other non-equilibrium phases. The questions remain regarding the types of preferred orientation under these kinetic and thermodynamic conditions.

In this study, the partial oxygen concentration was kept at $200 \mathrm{ppm}$ with a final heat treatment temperature ranged between $750{ }^{\circ} \mathrm{C}$ and $820^{\circ} \mathrm{C}$. Our experimental results have shown that most of the $a$-axis orientation takes place at 

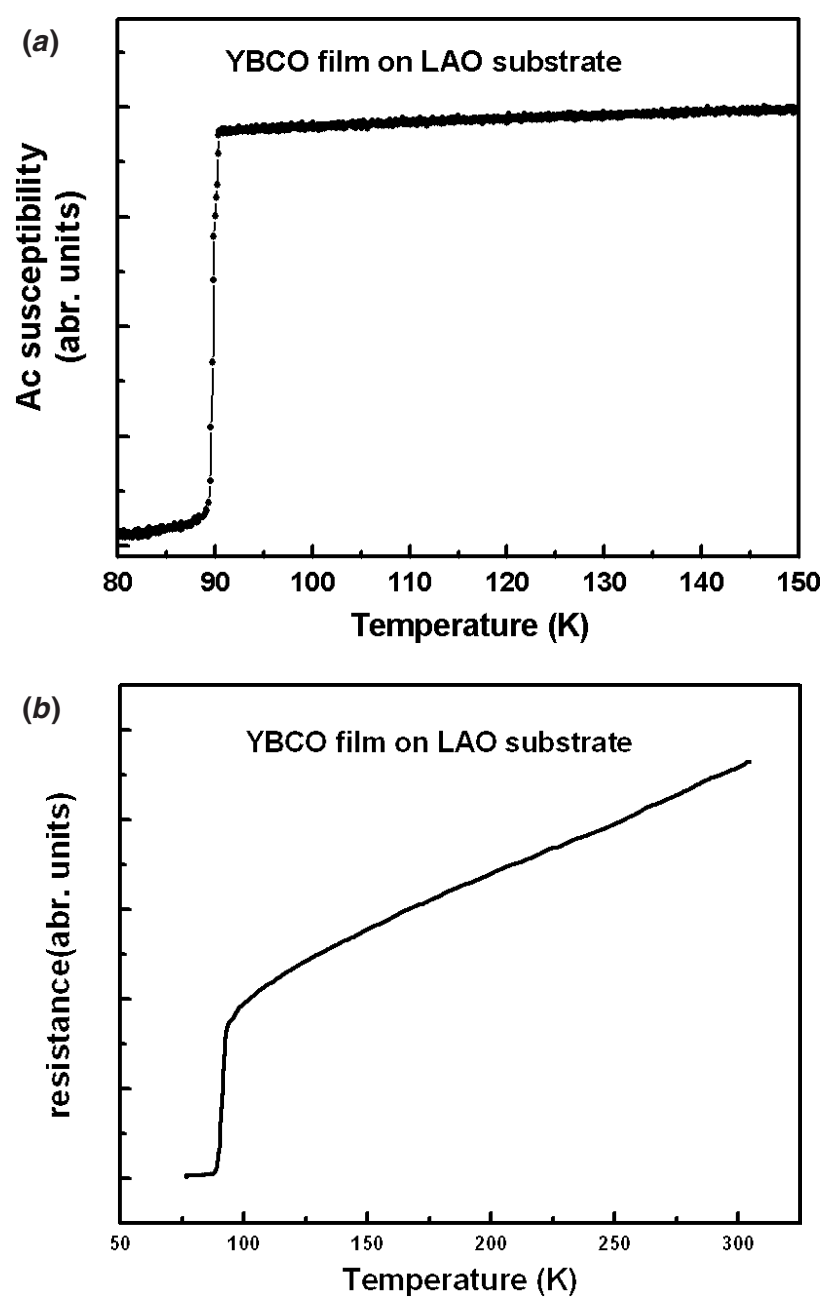

Figure 6. (a) ac susceptibility and (b) resistivity versus temperature for the $c$-axis oriented YBCO film.

relatively higher final heat treatment temperatures as evidenced in figure 4. Therefore, it appears that at a high temperature limit, the $a$-axis growth should be more energetically advantageous. The formation of YBCO grains from the amorphous-like precursor can be simulated to the situation of the crystallization process from a melt, which involves the diffusion-controlled nucleation and growth. Transformation will occur through local rearrangements in structure resulting from the random thermal motion of atoms in amorphous phase. Some of these local rearrangements will have the structure of YBCO phase, thus constituting embryos of YBCO phase. As seen from figure 7 , some of these embryos will be stable after their free energy change surmounts one critical value $\Delta G^{*}[21]$, constituting nuclei upon which YBCO can grow. Homogeneous nucleation can happen in the amorphous phase away from the substrate, and heterogeneous nucleation can happen coherently on the lattice match substrate, $\Delta G^{*}$ is higher for homogeneous nucleation than for heterogeneous nucleation. For a $c$-axis growth, the thermodynamic condition is critical that heterogeneous nucleation can happen stably, while the homogeneous nucleation is suppressed because of the higher $\Delta G^{*}$ needed. In this situation, the YBCO phase forms continuously on the heterogeneous nuclei, meanwhile, latent heat is released at the interface, modifying

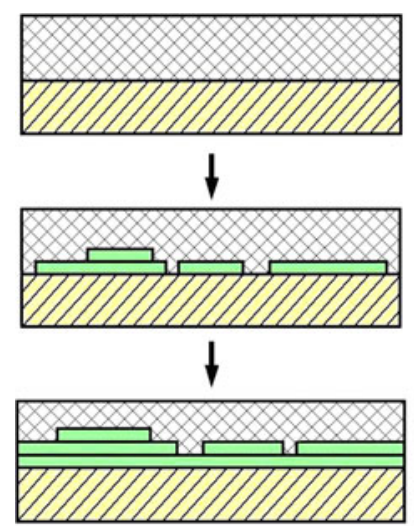

(a)

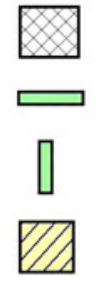

Figure 7. Schematic diagram showing (a) the layer-by-layer epitaxial $c$-axis grain growth and $(b)$ the $a$-axis texturing due to a higher driving force.

the temperature profile. Thus, the growth takes place only epitaxially on the substrate and the growth is advanced layer by layer while the amorphous precursor is kept in the nonequilibrium state, i.e. no nucleation would occur elsewhere. Figure 7(a) schematically illustrates this process.

For the epitaxial growth of YBCO film, this thermodynamic condition is sensitively controlled by a small temperature window $\Delta T$. Any disturbance or temperature variation exceeding this condition can result in other types of growth mechanism. In the TMAP synthesis, the commonly observed $a$-axis growth may fall into the latter mechanism. The $a$-axis orientation may take place under a high driving force condition due to enhanced YBCO crystallization kinetics. It has been reported that the growth rate along the $a$-axis $\left(R_{\mathrm{a}} \sim 0.66 \mu \mathrm{m} \mathrm{s}{ }^{-1}\right)$ is considerably higher than that along the $c$-axis $\left(R_{\mathrm{c}} \sim 0.54 \mu \mathrm{m} \mathrm{s}^{-1}\right)[22,23]$. At a lower driving force (lower temperature), the phase coexistence between YBCO and precursor is well balanced such that the layer-by-layer growth can be maintained in the entire heat treatment process. However, at higher driving force (higher temperature), both thermodynamic and kinetic conditions require a much faster crystallization. Then a more energetically favourable growth has to be taken in order to convert the precursor into the crystalline state. Since the $a$ axis growth assumes a fast rate advancing along the direction normal to the substrate, this would be a more advantageous growth pattern dominating the crystallization process. This process is schematically depicted in figure $7(b)$. As can be seen from figure $7(b)$, due to the lattice matching effect between the substrate and $c$-axis $\mathrm{YBCO}$, there is always one layer of $c$-axis grains, even at the condition which is favourable for $a$-axis growth. Meanwhile, the nucleation and growth of $a$-axis grains also happen away from the substrate in the 
Structural characterization of epitaxial YBCO thin films prepared by a fluorine-free sol-gel method for coated conductors
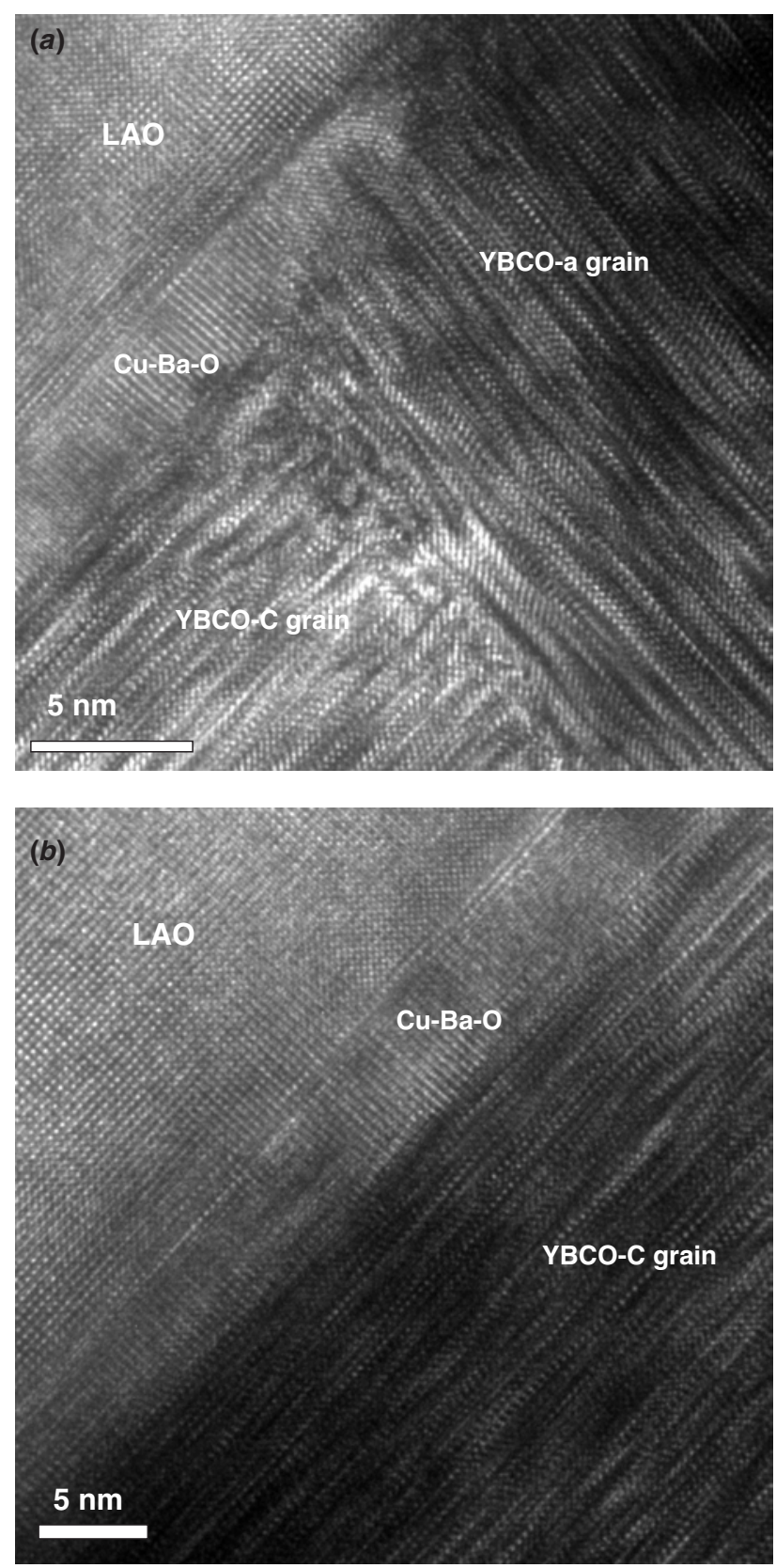

Figure 8. HRTEM images showing (a) $a$-axis grains directly form on the LAO substrate, and $(b)$ the interface second phase on which $c$-axis YBCO grains can grow.

amorphous phase, resulting in the abrupt contiguity between $a$ - and $c$-axis oriented grains in figures $4(a)$ and $(b)$.

It should be noted, in the previous studies of TFA deposited YBCO films, that well-grown $c$-axis grains were obtained at rather high firing temperatures $\left(900-920{ }^{\circ} \mathrm{C}\right.$ [1], 980-990 ${ }^{\circ} \mathrm{C}$ [7]). In some cases, the formation of $a$-axis oriented grains took place even at lower temperatures [4]. These results indicate quite different texturing mechanisms under these processing conditions. The kinetics of YBCO grain formation is related to the thermodynamic and structural conditions of the amorphous precursors. Due to fundamentally different synthesis routes and processing chemistry, the optimum firing temperature may vary in a wide range due to the stability of the amorphous state that is closely related to the defects and impurities. For instance, if there is a high density of pre-existing particles as impurities inside the amorphous phase, it is much easier to induce the heterogeneous nucleation at relatively low temperatures. We believe that the reaction chemistry of TFA is significantly different from that of TAMP. Therefore, direct comparison of these two synthesis routes will require detailed structural characterization of amorphous precursors and fully grown YBCO grains. Within these TMAP processing conditions introduced in this experiment, we found that the $a$-axis grains tended to form at the high temperature limit. This formation mechanism may be entirely different from those in the TFA films reported in previous studies $[1,4,7]$.

Besides the structural instability of the amorphous precursor being one of the core reasons for $a$ - and $c$-axis grain formation, other factors can also be important including impurity phases and reactions between $\mathrm{YBCO}$ and the substrate. As shown in figure 4(c), the boundary between $a$ - and $c$-axis grain exhibits amorphous and wavy-like features which are caused by lattice defects. However, these boundaries do not appear to have a significant effect on the nucleation and growth of both types of grains. We were also able to see, from the high-temperature treated film $\left(820^{\circ} \mathrm{C}\right)$, that some $a$-axis grains grow from the LAO substrate directly (figure $8(a)$ ), while the $c$-axis grains can form on a secondary phase (figure $8(b)$ ). The interface impurity shown in figure $8(b)$ is identified as $\mathrm{BaCuO}_{2}$ by both EDX spectrum and lattice imaging. Nonetheless, this secondary phase appears not important to the grain orientations.

Previous experimental results have indicated the effects of oxygen pressure and burnout temperature on texturing mechanisms. A general trend indicates that low oxygen pressure suppresses $a$-axis growth in the film. Although a detailed physical picture has not yet been established, it is clear that the enhanced cation diffusion due to low oxygen pressure can promote $c$-axis growth kinetics. High burnout temperature may result in precursor stability. This may further narrow the temperature window in which the $c$-axis growth is well controlled. Systematic study on the effects of these processing parameters is currently underway.

\section{Summary}

YBCO thin films were synthesized on LAO substrates by the TMAP fluorine-free solution method. While maintaining a low oxygen concentration at $200 \mathrm{ppm}$, the final heat treatment temperature was varied between $740{ }^{\circ} \mathrm{C}$ and $820{ }^{\circ} \mathrm{C}$ to study the texturing mechanism. It was found that the $c$-axis epitaxial growth tended to take place at low oxygen pressure, in agreement with the previous studies from the TFA films. The $c$-axis epitaxial growth was confirmed by HRTEM. A transport $J_{\mathrm{c}}$ on the order of $10^{6} \mathrm{~A} \mathrm{~cm}^{-2}$ at $77 \mathrm{~K}$ and zero field was previously reported on the YBCO films prepared by the same method. However, due to different precursor stability, the $c$-axis grains formed at relatively low temperatures. This result appears to be different from the previous TFA experiments. The $a$-axis texturing was often observed on films heat treated at high temperatures near $820^{\circ} \mathrm{C}$. HRTEM revealed structural lattice features of the $a$-axis oriented grains. Using the classic nucleation and growth model, the texturing mechanism was 
discussed from the point of view of a driving force for crystallization. At a high temperature, the $a$-axis texturing was a more sufficient and energetically favourable way of crystallization due to a faster growth rate along this direction.

\section{References}

[1] Gupta A, Jagannathan R, Cooper E I, Giess E A, Landman J I and Hussey B W 1988 Appl. Phys. Lett. 522077

[2] Chen Yen-Wei, Miyanaga Noriaki, Yamanaka Masanobu, Mitsuo Nakai, Tanaka Kazuo, Katsunobu Nishihara, Yamanaka Tatsuhiko and Nakai Sadao 1990 J. Appl. Phys. 681483

[3] Smith J A, Cima M J and Sonnenberg N 1999 IEEE Trans. Appl. Supercond. 91531

[4] Jee Y-A, Ma B, Maroni V A, Li M, Fisher B L and Balachandran U 2001 Supercond. Sci. Technol. 14285

[5] Goyal A, Norton D P, Budai J D, Paranthaman M, Specht E D, Kroeger D M, Christen D K, He Q, Saffian B, List F A, Lee D F, Martin P M, Klabunde C E, Hartfield E and Sikka V K 1996 Appl. Phys. Lett. 691795

[6] Araki T, Takahashi Y, Yamagiwa K, Iijima Y, Takeda K, Yamada Y, Shibata J, Hirayama T and Hirabayashi I 2001 Physica C 357-360 991

[7] Rice C E, van Dover R B and Fisanick G J 1987 Appl. Phys. Lett. 511842

[8] Gross M E, Hong M, Liou S H, Gallagher P K and Kwo J 1988 Appl. Phys. Lett. 52160

[9] Hamdi A H, Mantese J V, Micheli A L, Laugal R C O, Dungan D F, Zhang Z H and Padmanabhan K R 1987 Appl. Phys. Lett. 512152

[10] Peir-Yung Chu and Buchanan Relva C 1993 J. Mater. Res. 8 2134
[11] Gupta A, Cooper E I, Jagannathan R and Giess E A 1988 Chemistry of High-temperature Superconductors II ed D L Nelson and T F George (Washington, DC: American Chemical Society) p 265

[12] Shin-ichi Hirano, Takashi Hayashi and Miura Masashi 1990 J. Am. Ceram. Soc. 73885

[13] Parmigiani F, Chiarello G, Ripamonti N, Goretzki H and Roll U 1987 Phys. Rev. B 367148

[14] Yongli Xu, Shi Donglu, McClellen S, Buchanan R, Shixin Wang and Wang L M 2001 IEEE Trans. Appl. Supercond. 112865

[15] Donglu Shi, Yongli Xu, McClellan Shaun and Buchanan Relva 2001 Physica C 35471

[16] Donglu Shi, Yongli Xu, Wang S X, Lian J, Wang L M, McClellan Shaun M, Buchanan Relva and Goretta K C 2002 Physica C 37197

[17] Xu Y, Goyal A, Rutter N A, Shi D, Paranthaman M, Sathyamurthy S, Martin P M and Kroeger D M 2003 J. Mater. Res. 18677

[18] Chen Nan, Shi Donglu and Goretta K C 1989 J. Appl. Phys. 66 2485

[19] McIntyre P C, Cima M J and Roshko A 1995 J. Appl. Phys. 77 5263

[20] Castano O, Cavallaro A, Palau A, Gonzalez J C, Rossell M, Puig T, Sandiumenge F, Mestres N, Pinol S, Pomar A and Obradors X 2003 Supercond. Sci. Technol. 1645

[21] Owen F Devereux 1983 Topics in Metallurgical Thermodynamics (John Wiley \& Sons) p 429

[22] Ullrich M, Müller D, Krauns Ch, Bringmann B, Leenders A, Brandt C, Reeder M, Preusser A and Freyhardt H C 1997 International Workshop on Superconductivity, Hawaii p 76

[23] Nishizono K et al 1998 J. Japan. Inst. Metals 62562

[24] Zhao B, Yao H B, Shi K, Han Z H, Xu Y L and Shi D L 2003 Physica C 386348 